\title{
ON MINIMAL SEPARATING COLLECTIONS
}

\author{
BEZALEL PELEG ${ }^{1}$
}

1. Introduction. Minimal separating collections were introduced in $[2, \S 8]$; their knowledge is useful for the computation of the kernel of a cooperative game. In this note we determine an exact bound on the maximum number of sets which a minimal separating collection can have. The proof makes use of a result on finite graphs, which is proved in the next section.

2. Minimally ordered graphs. Throughout this paper we deal only with directed graphs. Our terminology is that of [1]. We recall some definitions that pertain to our work. A finite graph $G$ is a pair $(X, \Gamma)$, where $X$ is a finite set and $\Gamma$ is a multivalued function mapping $X$ into $X$, i.e., for each $x \in X, \Gamma(x)$ is a subset of $X$. Let $G=(X, \Gamma)$ be a finite graph. An element $x$ of $X$ is called a vertex. The number of vertices of $X$ is denoted by $n$. An ordered pair of vertices $(x, y)$ with $y \in \Gamma(x)$ is an arc. A path is a sequence of vertices $\mu=\left[x_{1}, \cdots, x_{k+1}\right]$ such that $x_{i+1} \in \Gamma\left(x_{i}\right)$ for $i=1, \cdots, k$. Let $\mu=\left[x_{1}, \cdots, x_{k+1}\right]$ be a path. $\mu$ is a circuit if $x_{1}=x_{k+1}$; if, in addition, $x_{j} \neq x_{i}$ for $i \neq j, 1 \leqq i, j \leqq k$, then $\mu$ is an elementary circuit. $G$ is strongly connected if for every ordered pair of distinct vertices $x$ and $y$ there exists a path $\mu=\left[x, a_{1}, \cdots, a_{k-1}, y\right]$. A partial graph $G^{\prime}$ of $G$ is a graph $\left(X, \Gamma^{\prime}\right)$, where $\Gamma^{\prime}(x) \subset \Gamma(x)$ for every $x \in X ; G^{\prime}$ is a proper partial graph of $G$ if there exists a vertex $y$ such that $\Gamma^{\prime}(y)$ is a proper subset of $\Gamma(y)$. Let $A$ be a subset of $X$. The subgraph of $G$ determined by $A$ is the graph $\left(A, \Gamma_{A}\right)$ where $\Gamma_{A}(a)$ $=\Gamma(a) \cap A$, for all $a \in A$. An $s$-graph is a set $Y$ together with a collection $U$ of ordered pairs of members of $Y$; $U$ may contain the same ordered pair as many as $s$ times. Clearly a 1-graph is a graph. The shrinkage of $A$ is the $s$-graph obtained from $G$ by deleting the arcs of the subgraph $\left(A, \Gamma_{A}\right)$, and by identifying all the vertices of $A$. The number of members of $A$ is denoted by $|A|$. Let $x$ and $y$ be vertices of $G$. We write $x \leqq y$ if $x=y$ or if there exists a path $\mu=\left[x, a_{1}, \cdots, a_{k-1}, y\right]$. The relation $\geqq$ is the weak ordering associated with $G$. We write $x<y$ if $x \leqq y$ but not $x \geqq y$; we write $x \equiv y$ if $x \leqq y$ and $y \leqq x$. The relation $\equiv$ is the equivalence relation derived from $\leqq$.

Received by the editors October 1, 1966.

1 The research described in this paper was partially supported by the United States Office of Naval Research, under Contract No. N62558-4355, Task No. NR 047-045. Reproduction in whole or in part is permitted for any purpose of the United States Government. 
Definition 2.1. A finite graph $G$ is minimally ordered if it has no proper partial graph which defines the same weak ordering on the vertices of $G$ as $G$.

Clearly a minimally ordered strongly connected graph is minimally connected [1, p. 123].

Lemma 2.2. A subgraph of a minimally ordered graph is minimally ordered.

The proof, which is straightforward, is omitted.

Lemma 2.3. Let $G$ be a minimally ordered graph and let $A \subset X$ determine a strongly connected subgraph; the shrinkage of $A$ leads to a minimally ordered graph.

The proof, which is similar to the proof of Theorem 1 [1, p. 123], is omitted.

Lemma 2.4. A minimally connected graph $G$ has at most $2(n-1)$ arcs.

Proof. The proof is by induction on $n$. For $n=1$ the lemma is true. Let $G$ be a minimally connected graph with $n$ vertices, $n \geqq 2$. $G$ contains an elementary circuit $\mu$ with $l$ vertices, $l \geqq 2$. By Theorem 1 $[1$, p. 123], the shrinkage of $\mu$ leads to a minimally connected graph $G^{\prime}$. $G^{\prime}$ has $n-l+1$ vertices. Since $n-l+1<n, G^{\prime}$ has, by assumption, no more than $2(n-l)$ arcs. By Theorem $2[1$, p. 124] $G$ has at most $2(n-l)+l=2 n-l \leqq 2 n-2$ arcs.

ExAmple 2.5. Let $X$ be a set with $n$ members, $n \geqq 1$, and let $a \in X$. Define $\Gamma(a)=X-\{a\}$ and $\Gamma(x)=\{a\}$, for $x \in X-\{a\} .(X, \Gamma)$ is a minimally connected graph with $2(n-1)$ arcs.

Lemma 2.6. A minimally ordered graph without circuits has at most $f(n)$ arcs, where $f(n)=\frac{1}{4} n^{2}$ if $n$ is even, and $f(n)=\frac{1}{4}\left(n^{2}-1\right)$ if $n$ is odd.

Proof. The proof is by induction on $n$. For $n=1$ the lemma is true. Let $G=(X, \Gamma)$ be a minimally ordered graph without circuits with $n$ vertices. We shall assume that $n$ is even. The proof for odd $n$ is similar. Let $a$ be a minimal vertex of $G$, i.e., there exists no $x \in X$ such that $a>x$. Let $T=\{b \mid b>a$ and there exists no $c$ such that $b>c>a\}$. We remark that $x \in \Gamma(a)$ if and only if $x \in T$. If $|T| \leqq \frac{1}{2} n$ let $X_{1}$ $=X-\{a\}$. If $|T|>\frac{1}{2} n$ let $b \in T$ and $X_{1}=X-\{b\}$. We remark that the number of arcs incident into or out from $b$ is less than $\frac{1}{2} n$, since if $b_{1} \in T$ then neither $b_{1}>b$ nor $b>b_{1}$. By Lemma 2.2 the subgraph determined by $X_{1}$ is minimally ordered; since it has no circuits it has, by assumption, at most $f(n-1)$ arcs. Hence $G$ has no more than $f(n-1)+\frac{1}{2} n=f(n)$ arcs. 
Example 2.7. Let $X$ be a set with $n$ members, $n \geqq 1$. Let $A \subset X$ have $\frac{1}{2} n$ members if $n$ is even, and $\frac{1}{2}(n+1)$ members if $n$ is odd. Let $\Gamma(a)=X-A$, for $a \in A .(X, \Gamma)$ is a minimally ordered graph without circuits which has $f(n)$ arcs.

Lemma 2.8. A minimally ordered graph $G=(X, \Gamma)$ has at most $g(n)$ arcs, where $g(n)=\max (2(n-1), f(n))$.

Proof. Let $X_{1}, \cdots, X_{k}$ be the equivalence classes determined by the equivalence relation derived from the weak ordering associated with $G$. By Lemma 2.2 , for $i=1, \cdots, k,\left(X_{i}, \Gamma_{X_{i}}\right)$ is a minimally connected subgraph of $G$. If $k=n$ then $G$ has no circuits. Thus if $k=1$ or $k=n$ the lemma is true by Lemmas 2.4 and 2.6. If $2 \leqq k \leqq n-1$ then, by Lemma 2.3 , the shrinkage of $X_{1}, \cdots, X_{k}$ leads to a minimally ordered graph $G^{*}$. Clearly $G^{*}$ has no circuits; hence it has no more than $\frac{1}{4} k^{2}$ arcs. By Lemma 2.4 each of the subgraphs $\left(X_{i}, \Gamma_{\boldsymbol{X}_{\boldsymbol{i}}}\right)$, $i=1, \cdots, k$, has at most $2\left(\left|X_{i}\right|-1\right)$ arcs. Thus $G$ has no more than

$$
\frac{1}{4} k^{2}+2 \sum_{i=1}^{k}\left(\left|X_{i}\right|-1\right)=\frac{1}{4} k^{2}+2(n-k) \leqq g(n)
$$

arcs.

COROLlaRy 2.9. Every finite graph $G$ has a partial graph with no more than $g(n)$ arcs which defines the same weak ordering on the vertices of $G$ as $G$.

3. Minimal separating collections. We recall some of the definitions of [2]. Let $N$ be a finite nonempty set. Let $D$ be a collection of subsets of $N$ and let $i, j \in N, i \neq j . i$ is separated by $\mathscr{D}$ from $j$ if there exists a set $S \in D$ such that $i \in S$ and $j \notin S$. D is separating if for every pair $i, j \in N, i$ is separated from $j$ by $D$ if and only if $j$ is separated from $i$ by $D$. A separating collection is minimal separating if it does not contain a proper subcollection which is separating. A collection $D$ is completely separating if for all pairs $i, j \in N, i \neq j, i$ is separated from $j$ and $j$ is separated from $i$ by $D$. A completely separating collection is minimal completely separating if it does not contain a proper subcollection which is completely separating. We remark that a completely separating collection which is minimal separating is a minimal completely separating collection.

ExAMPLE 3.1. Let $N$ be a finite nonempty set. The collections $D_{1}(N)=\{\{i\}: i \in N\}$ and $D_{2}(N)=\{N-\{i\} \mid i \in N\}$ are minimal separating and completely separating.

EXAMPLE 3.2. Let $N=\{1, \cdots, n\}$ be the set of the first $n$ natural numbers. Let $S_{i}=\{j \mid j \leqq i\}, \quad i=1, \cdots, n-1$. The collection 
$\left\{S_{1}, \cdots, S_{n-1}, N-S_{1}, \cdots, N-S_{n-1}\right\}$ is minimal completely separating and has $2(n-1)$ sets.

Example 3.3. Let $N$ be a set with $n$ members, $n \geqq 5$. Let $A \subset N$ have $\frac{1}{2} n$ members if $n$ is even, and $\frac{1}{2}(n-1)$ members if $n$ is odd. The collection $\left\{S \cup T \mid S \in D_{1}(A), T \in D_{2}(N-A)\right\}$ is minimal separating and completely separating and has $f(n)$ sets (see Example 3.1, where $D_{1}$ and $D_{2}$ are defined, and Lemma 2.6 where $f(n)$ is defined).

DEFINITION 3.4. Let $D$ be a collection of subsets of a finite nonempty set $N$. An ordered pair $(i, j)$ of members of $N$ is a distinguished pair (with respect to $D$ ) if there is exactly one set $S \in \mathbb{D}$ such that $i \in S$ and $j \notin S$.

LEMMA 3.5. If $D$ is a minimal completely separating collection of subsets of a finite nonempty set $N$, then for each $S \in D$ there exists a distinguished pair $(i, j)$ such that $i \in S$ and $j \notin S$.

The proof, which is straightforward, is omitted.

Theorem 3.6. Let $N$ be a finite nonempty set with $n$ members. The maximum number of sets in a minimal completely separating collection of subsets of $N$ is $g(n)$ (see Lemma 2.8 where $g(n)$ is defined).

Proof. Let $D$ be a minimal completely separating collection of subsets of $N$. Define a graph $G=(N, \Gamma)$, where $\Gamma$ is defined by: $j \in \Gamma(i)$ if and only if $(i, j)$ is a distinguished pair (with respect to D). By Corollary 2.9, $G$ has a partial graph $G^{\prime}=\left(N, \Gamma^{\prime}\right)$ which defines the same weak ordering as $G$ and has no more than $g(n)$ arcs. Let $D^{\prime} \subset D$ be defined by: $\mathscr{D}^{\prime}=\{S \mid S \in \mathscr{D}$ and there exists a pair $(i, j)$ such that $j \in \Gamma^{\prime}(i), i \in S$ and $\left.j \notin S\right\}$. $\mathscr{D}^{\prime}$ has at most $g(n)$ sets. We shall show that $D^{\prime}=\mathbb{D}$. Suppose, per absurdum, that there exists $S \in \mathbb{D}-D^{\prime}$. By Lemma 3.5 there exists a distinguished pair $(i, j)$ such that $i \in S$ and $j \notin S ; j \geqq i$ according to the ordering of $G$. Since $G^{\prime}$ defines the same ordering, there exists a path $\mu=\left[i, a_{1}, \cdots, a_{l-1}, j\right]$ in $G^{\prime}$. Let $S_{1}, \cdots, S_{l}$ be sets in $D^{\prime}$ such that $i \in S_{1}, a_{1} \notin S_{1}, a_{1} \in S_{2}, a_{2} \notin S_{2}, \cdots$, $a_{l-1} \in S_{l}, j \notin S_{l}$. Since $i \in S, S \neq S_{1}$ and $\left(i, a_{1}\right)$ is a distinguished pair, $a_{1} \in S$. Using induction we can show that all the vertices of $\mu$ are in $S$. Since $j \notin S$ we have a contradiction which shows that $\mathscr{D}^{\prime}=\mathscr{D}$. Hence the number of sets in $D$ is not greater than $g(n)$. Examples 3.2 and 3.3 show that the bound $g(n)$ is attained.

Corollary 3.7. Let $N$ be a finite nonempty set with $n$ members. The maximum number of sets in a minimal separating completely separating collection of subsets of $N$ is $f(n)$ for $n \geqq 7$, and is not greater than $2(n-1)$ for $n<7$. 
Proof. Theorem 3.6 and Example 3.3.

\title{
REFERENCES
}

1. C. Berge, The theory of graphs and its applications, Wiley, New York, 1962.

2. M. Maschler and B. Peleg, A characterization, existence proof and dimension bounds for the kernel of a game, Pacific J. Math. 18 (1966), 289-328.

The Hebrew University of Jerusalem

\section{IMBEDDING CLOSED RIEMANN SURFACES IN $C^{n}$}

\author{
K. V. RAJESWARA RAO ${ }^{1}$
}

I. Introduction. Let $R$ be a closed Riemann surface of genus $g$, $G$ a nonempty, open subset of $R$, and $A$ the set of all complex valued functions that are continuous on $R$ and holomorphic on $G$. With the usual pointwise operations $A$ is an algebra over the complex field. We consider the problem: how many functions in $A$ suffice to separate points of $R$ ?

Let $f$ be a nonconstant member of $A$. If the genus $g=0$, Wermer [4] showed that there exist $f_{1}$ and $f_{2}$ in $A$ which, together with $f$ separate points of $R$; if $g=1$, Arens [2] established the existence of $f_{1}, f_{2}$ and $f_{3}$ in $A$ which, together with $f$ separate points of $R$. In this note we shall present a modification of the Wermer-Arens argument to prove the following

TheOREM. Let the genus $g$ be arbitrary. If A contains nonconstant functions, then there exist four functions in $A$ which separate points of $R$ and which have no common branch points in $G$.

The author is indebted to a referee for pointing out an error in an earlier version of the paper.

II. Two lemmas. Let $\phi$ be a nonconstant member of order $n$ in the field $K$ of meromorphic functions on $R$. Let $w$ be a point of the extended plane which has $n$ distinct inverse images under $\phi$. Denote by $E(\phi, w)$ the finite set which is the union of $\phi^{-1}(w)$ and $\phi^{-1}(\phi(b))$ as $b$ ranges over all the branch points of $\phi$. For (fixed) $\phi$ and $\psi$ in $K$, let $S$

Received by the editors December 7, 1966.

${ }^{1}$ Supported by the Air Force Office of Scientific Research and the Purdue Research Foundation. 